\title{
Structure Augmented Monocular Saliency for Planetary Rovers
}

\author{
Conrad Spiteri ${ }^{1}$, Affan Shaukat, Yang Gao \\ S.T.A.R. Lab. \\ Surrey Space Centre, University of Surrey, Guildford, Surrey, GU2 7XH, UK
}

\begin{abstract}
This paper proposes a novel object detection method based on the visual saliency model in order to reliably detect objects such as rocks from single monocular planetary images. The algorithm takes advantage of the relatively homogeneous and distinct albedos present in planetary environments such as Mars or the Moon to extract a Digital Terrain Model of a scene using photoclinometry. The Digital Terrain Model is then incorporated into a bottom-up visual saliency algorithm to augment objects that protrude out of the ground. This Structure Augmented Monocular Saliency algorithm (SAMS) improves the accuracy and reliability of detecting objects in a planetary environment with no training requirements, greater robustness and lower computational complexity than 3D saliency models. Comprehensive analysis of the proposed method is performed using three challenging benchmark datasets. The results show that the Structure Augmented Monocular Saliency (SAMS) algorithm performs better than against commonly used visual saliency models on the same datasets.
\end{abstract}

Keywords: Planetary rovers, object detection, visual saliency

\section{Introduction}

Over the past few decades extra-terrestrial planetary rovers have evolved into highly complex intelligent systems utilising a variety of on-board sensors.

\footnotetext{
${ }^{1}$ Corresponding author e-mail: c.spiteri@surrey.ac.uk
} 
In particular, machine vision has played an important role in increasing rover autonomy. Visual feature detection remains a key research topic, especially for rover localisation, pose estimation and navigation. To date, most of these planetary visual navigation systems incorporate the well-known technique of saturated feature extraction, using basic point-based feature detection methods (e.g., SURF features). There is a clear understanding that there is still much work that remains to be done to provide algorithms that can detect landmarks on extraterrestrial planetary surfaces [1. Moreover identification of landmarks such as, rocks in terms of point-based features (SIFT, SURF and corner-based) using supervised classification techniques are computationally intensive, and prone to failures due to homogeneous surfaces textures of Mars. Furthermore, image databases for training purposes (from previous missions) are limited, and may over fit models to very specific terrain patterns that are not generic. Thus, it is important for landmarks, such as rocks, to be detected using methods that are generic, unsupervised, and do not rely on visual descriptors that are computationally intensive.

The use of stereopsis (or stereovision) has been the most widely used solution for terrain mapping on Mars. However there will be a greater requirement for identification of terrain features, such as rocks, slopes and other related hazards as landmarks for future extra-terrestrial missions. Where stereovision and active LiDAR-based solutions do exist in terrestrial rovers for such applications [2, 3, 4, they might prove to be computationally intensive for planetary rovers. Alternatively, the use of monocular vision-based techniques may prove to be a more optimal solution in terms of hardware, software and power consumption. There is an increasing understanding that monocular vision-based object detection methods offer great potential for the identification of landmarks on planetary surfaces. Most popular methods include; shape analysis and detection, edge-detection operators, interest-point detectors, and Haar-like features [5. However, these features may exhibit suboptimal performance if training data is not sufficiently large.

On the other hand, monocular vision processing does not necessarily require 
as much computational power as stereo vision processing. Furthermore it can be used to detect objects at a much further distance than stereo vision processing 5]. Being able to identify long-range obstacles and other terrain parameters is a relatively new area of research, whose results will be of clear relevance for planetary explorations.

For more than a decade, there has been an effort to develop machine vision paradigms that can extract regions of interest (ROIs) in terms of their global and local conspicuity characteristics, known as visual saliency models 6]. To date, one such method, i.e., Itti et al. [7, has been used for the detection of planetary rocks [8] within the context of planetary rovers. However, a more in depth investigation is required into such models, in order to identify the most feasible stimuli for such planetary mission applications. This paper investigates an object detection technique dubbed Structure Augmented Monocular Saliency (SAMS) in order to reliably detect objects (e.g., rocks) from a sequence of planetary $2 \mathrm{D}$ images. The proposed solution is based on existing state-of-the-art saliency algorithms and augmented using a rough Digital Terrain Model of the scene acquired using photoclinomentry (or Shape from Shading) from the same monocular image. This technique seeks to abate some algorithmic limitations of existing methods without a-priori model training requirements, lower computational complexity and greater robustness towards applications over long-distance planetary terrains. The proposed method is a key component of the Planetary Monocular Simultaneous Localization and Mapping (PM-SLAM) algorithm [9, however there are various applications for which the SAMS algorithm can be employed. Typical usage scenarios are discussed in detail in [10]

The rest of the paper is organized as follows: Section 2 reviews and analysis cognitive-inspired" visual saliency-based methods for rock detection as landmarks with the potential for application to the problem of autonomous rover navigation and absolute localisation. Section 3 discusses the state-of-the-art behind Shape from Shading. Section 4 discusses the SAMS algorithm and depicts the test results by comparing them to our previous studies, while our conclusions are presented in Section 5. 


\section{Visual Saliency in Planetary Environments}

Visual saliency (or more generally visual attention) models are mostly inspired by the information selection property of biological visual systems and their underlying paradigms can either be based on computational models or cognitive research. Applications of visual saliency models cover a range of different areas; from low-level object detection and tracking [11, 12, to more complex robot localisation and navigation [13. Models of visual attention can vary on the basis of their processing characteristics, they can either take top-down factors into account (that relate to high-level cognitive factors) or bottom-up (called saliency") processing [6]. We specifically focus on the bottom-up attention models. A general understanding about natural top-down attentional behaviour can be attained from [14. Further classifications exist in literature, such as object-based i.e., prediction of salient regions is based on detection and segmentation of objects in the visual scene or space-based (i.e., notion of saliency is based at pixel-level in probabilistic terms of attracting attention [6].

Within the context of the current problem domain we will focus on models that are bottom-up, space-based and are able to generate topographic saliency maps of the input visual scene. Further to this, we select models that are relatively fast among the state-of-the-art and have very low computational load.

We select seven state-of-the-art saliency models for the current object detection and tracking problem and perform extensive comparative analysis using standard evaluation protocols. The choice of saliency models for the current research problem is constrained by the visual feature characteristics of Martian terrain. Furthermore limitations in power and computational resources on-board planetary rovers has influenced the selection of saliency models for the potential application environment, and therefore algorithms with the lowest possible computational load have been used. More importantly, vision-based methods that require prior training (supervised learning) may not be appropriate for planetary rovers, since access to data from previous missions is either very limited or insufficient for training and testing purposes. 
The work by Itti et al. 7] (Itti-98) was the forefront in bottom-up visual saliency modelling that relates to human visual search strategies. It uses centresurround differences across multi-scale image features within three topographical feature maps (colour, intensity and orientation) for identifying conspicuous regions. The three feature maps are combined into a single saliency map for local conspicuity over the entire visual scene. Walther et al. 15] (STB (Saliency Toolbox)) extends this concept towards modelling visual attention in terms of proto-objects (the generated saliency maps are used for the deduction of a proto-object (via a winner-take-all neural network framework) at the attended location), (see [16, 17 for further literature on proto-objects). Harel et al. [18] (GBVS) uses the computational power, topographical structure, and parallel nature of probabilistic graphical models in order to describe visually salient regions in an image. The equilibrium distribution of Markov chains along with a dissimilarity measure is used to compute saliency values in the visual scene. Hou et al. 19] (SRA) proposed the use of log spectrum of an input image along with the average Fourier envelope in order to extract the spectral residual in the frequency domain to generate a saliency map. Seo et al. [20] (SDSR) proposed a unified framework for (static and space-time) saliency detection that defines visually conspicuous regions in a local way. The model utilises non-parametric local regression kernels to estimate the likelihood of pixel to its surrounding. A self-resemblance map (that is used to estimate saliency likelihood) is generated which measures the statistical likelihood (similarity) of a feature matrix at a given pixel to its surround feature matrices using matrix cosine similarity measure. Guo et al. 21] (PQFT) introduced a multi resolution spatiotemporal based visual saliency detection model called phase spectrum of quaternion Fourier transform (PQFT) that represents an image in terms of colour, intensity, and motion features (texture pop-out). This model captures the temporal characteristic (an additional motion dimension) of conspicuous regions within a visual scene in addition to its spatial conspicuity and has been proven to have very low computational complexity with little compromise on performance. Hou et al. 22] (SigSal ) model is based on the concept of figure-ground separation for 
separating objects from the background using a binary, holistic image descriptor, called image signature (performed within the framework of sparse signal mixing).

The selection of visual saliency models discussed above manifest conspicuous regions of interest in the image using distinct recognition paradigms. Each individual technique seems to fit the current problem domain. We will set out to quantify which of these visually salient feature modelling methods is the most suitable for rock detection on a homogeneous planetary surface with the potential for autonomous planetary exploration missions. Knowledge gained form the current analysis will prove to be very useful for the development of biologically inspired models especially adapted to homogeneous planetary surfaces such as the Structure Augmented Monocular Saliency (SAMS) algorithm discussed in the following section.

\section{Depth Perception}

The human visual search strategy is based on the feature integration theory [7] of a biologically plausible architecture as proposed in [23. The visual input from the eyes is first decomposed into a set of topographic feature maps. The different maps then compete for saliency within the final map where prominent features stand out from their surroundings producing a final saliency map.

With the advent of stereo vision cameras and increased processing power available at lower costs, saliency models that incorporate depth cues have emerged in literature, however, computing depth from disparity is a computationally expensive process $[24,25,26$, that planetary rovers can ill afford [27, which makes the algorithm inept at operating in real-time due to limited processing power available on planetary rovers [28, 29]. Photoclinometry or shape from shading (SfS) is a cluster of techniques for estimating topography that have been exploited by planetary scientists for more than 50 years [30. The techniques fall under two main classes, global methods and local methods.

Global methods minimise a cost function involving constraints such as smooth- 
ness and integrability to recover the surface normal gradient or depth through variation calculus. They iteratively compute the shape which is globally consistent. Global methods tend to be more complex but produce better results than local methods [31. Local methods on the other hand are simpler but only provide an approximation of the shape.

In SfS, it is assumed that the reflectance map is known and the albedo map is homogeneous or linear. Within terrestrial images the latter may not always be the case since albedos of objects around us manifest diverse variations in a non-linear form. Although it is somewhat possible to extract an albedo map from a single image without any prior knowledge, results tend to vary based on the constraints and assumptions within the study [32. In contrast, dusty environments such as the Moon and Mars tend to manifest a limited range of albedos with distinct variations [33, 34] that are easily deducible and quantifiable [32, 35].

SfS algorithms are fundamentally based on Lambert reflectance function and cosine law [32, where local methods employ discrete approximations for $\mathrm{p}$ and q using finite differences and linearization of the reflectance map. Tsai and Shah 32 provide a good comparison between their method and other local SfS algorithms resulting in one of the fastest SfS methods available. Using a Jacobian iterative method they reduce a complex calculation to equation 1 by solving part of the equation using previous estimates and assuming the initial state of the previous estimate at the first iteration to be zero.

$$
\left.Z_{(}(x, y)\right)^{n}=Z_{(x, y)}^{n-1}+\frac{-f Z_{(x, y)}^{n-1}}{\frac{d}{d Z(x, y)} f Z_{(x, y)}^{n-1}}
$$

where

$$
\begin{gathered}
\frac{d f\left(Z_{(x, y)}^{n-1}\right)}{d Z_{(x, y)}}=-1\left(\frac{p_{s}+q_{s}}{\sqrt{p^{2} q^{2}+1} \sqrt{p_{s}^{2} q_{s}^{2}+1}}-\frac{(p+q)\left(p p_{s}+q q_{s}+1\right)}{\sqrt{\left(p^{2} q^{2}+1\right)^{3}} \sqrt{p_{s}^{2} q_{s}^{2}+1}}\right) \\
p=\frac{\delta Z}{\delta x^{\prime}} \quad q=\frac{\delta Z}{\delta y^{\prime}} \quad p_{s}=\frac{\cos \tau \sin \sigma}{\cos \sigma} \quad q_{s}=\frac{\sin \tau \sin \sigma}{\cos \tau}
\end{gathered}
$$

$\tau$ and $\sigma$ are the tilt and slant angle of the illumination source. 
The authors of 32] also extend the algorithm to specular surfaces and tested

algorithms are benchmarked for speed and accuracy with the Tsai and Shah algorithm described above having the fastest execution speed with reasonable accuracy. Therefore the Tsai and Shah algorithm has been chosen to extract the approximate structure of the scene.

As described by Ramachandran [37 the ability to perceive shape (or structure) from shading is one of the most important yet poorly understood aspects of human vision. In addition recovering structure from shading involves low computational costs and hence is ideally suited for planetary rovers.

\section{The SAMS Algorithm}

\subsection{Saliency Object Detection}

Intensity based weightings are associated with multiple clusters of pixels using the aforementioned methods; outlining the regions of interest (ROI) in the visual scene. These saliency blobs with non-strict boundaries need to be converted into binary saliency maps, i.e., ROI blobs with hard boundaries segregating them from the background using an intensity threshold selection criterion. The resulting binary blobs can serve as semantic representation of important surficial rocks in the input image that are used as landmark features (with the ultimate goal of rover localisation, pose estimation and navigation). Surface rocks are therefore completely described on the basis of their local or global conspicuity pop-out characteristics. An automatic threshold selection criterion is applied to the saliency map in order to convert it to a binary map. We use Otsu's method [38] (histogram shape-based thresholding) to reduce our saliency map to a binary image with the assumption that the saliency maps have a bimodal distribution, (i.e., two classes of pixels; salient objects (i.e., rocks) and the background). This method essentially follows an exhaustive search strategy (discriminant form of pattern recognition technique) to compute the optimum 
threshold that minimises the intra-class variance or maximising the inter-class variance,

$$
\sigma_{B}^{2}=\rho_{1}(\mathcal{T}) \rho_{2}(\mathcal{T})\left(\mu_{1}(\mathcal{T})-\mu_{2}(\mathcal{T})\right)^{2}
$$

where $\rho_{1}(\mathcal{T})$ and $\rho_{2}(\mathcal{T})$ are the probabilities of these two classes $\left(C_{1}\right.$ and $\left.C_{2}\right)$ respectively, and

$$
\rho_{1}(\mathcal{T})=P_{r}\left(C_{1}\right)=\sum_{i=1}^{\mathcal{T}} p(i), \quad \rho_{2}(\mathcal{T})=P_{r}\left(C_{2}\right)=\sum_{i=1}^{\mathcal{T}+1} p(i)
$$

and $\mathcal{T} \in\{1,2,256\}$ represents any level within the full range of grey level histogram values. In most cases, the Otsu's 38 method iteratively computes the optimum by maximising (2), more formally,

$$
\sigma_{B}^{2}\left(\eta^{*}\right)=\underset{1<\eta<\mathcal{K}}{\operatorname{argmax}} \sigma_{B}^{2}(\eta)
$$

This banalization step further acts as a quasi post-processing-filter in order to suppress the response of sporadic saliency blobs in the image, while nonetheless allowing for the possibility of detection failures.

\subsection{Pre-processing and Horizon Detection}

A horizon (sky) detection method has been employed as a pre-process, outside the saliency algorithms, to crop all images just below the sky line as this interferes with the resultant output. Sky detection is performed by searching for a large and bright homogeneous region that touches at least one of the four corners of the image. It is possible for a sky region not to include at least one of the four corners of the image, when a corner is occluded by another object (e.g. part of the rover or a hilly outcrop), however this does not manifest itself in the chosen dataset. The sky detection starts with building an edge map. Candidate contours are extracted using the border following method of Suzuki [17. Contours that do not encapsulate any of the four corners are rejected. Among the remaining candidates, the brightest homogeneous patch, based on standard deviation is assumed to contain the sky region. The lowest sky point 
from all the images determines the crop height and all images are cropped at this level in order to conserve the consistency of image dimensions throughout the dataset and positions of the annotated rocks.

Furthermore, some images manifest vignetting distortion. Vignetting refers to the phenomenon of reduced intensity at the periphery of the image. Although it may seem negligible to the average viewer or most computer vision algorithms, it can significantly impair SfS algorithms from performing correctly as they rely on precise intensity data. This distortion is corrected as a pre-process, outside the saliency algorithms, in a similar manner to [39]. The SAMS algorithm is able to process images from a very broad spectrum of detector sizes, angular resolutions, Field of views and Signal to Noise ratios with no or minimal rectification required.

\subsection{Datasets}

\subsubsection{SSC Lab-based Dataset}

The dataset was recorded in our research lab at the Surrey Space Centre, University of Surrey. It involves a rover (with four wheels, (Pioneer)), equipped with a range of sensors, traversing a flat surface littered with scattering of rock clusters.

The rover is tele operated to conduct oval shaped circuits at low speeds between coarse-grained parallel formation of rock clusters in order to simulate both straight and curved path manoeuvres. The flat surface 2D manoeuvres mitigate some level of complexity resulting from effects of elevation in a 3-D traversal (commonly experienced by planetary exploration rovers).

This provides us with an intermediate step towards validation process of vision based algorithms. The collected dataset consists of low-level features including odometry data (with an accuracy of $1 \mathrm{~mm}$, although prone to errors causing drift in pose estimates) of the rover's pose ('heading', 'yaw') deduced from the on-board shaft encoders and external video scene capture using a single on-board camera mounted with a known reference frame relative to the rover's pose. 
The dataset comprises a total of 230 frames (per frame monocular image size of $640 \times 480$ pixels, at 15-fps sampling). Refer to figure 3 a

\subsubsection{PANGU Dataset}

The dataset was generated using a combination of the Planet and Asteroid Natural scene Generation Utility (PANGU), (developed at the University of Dundee) and image capture software from the PMSLAM project at the SSC. PANGU simulates planetary environments using parameters such as the levelness of the terrain and the number, size and distribution of craters and boulders. The PMSLAM software has the ability to place a virtual camera at a given location, field of view and record images. The dataset comprises a total of 111 frames (per frame monocular image size of 512 x 512 pixels). Refer to figure $3 \mathrm{~b}$

\subsubsection{RAL Space SEEKER Dataset}

The dataset is a subset of the original data generated by the SEEKER consortium. It consists of rectified monocular images from the left camera (a Bumblebee XB3) of a sensor equipped rover (providing additional low-level sensor data, such as, DGPS, IMU Data, DTM, VO and Path data). The experimental dataset comprises a total of 111 frames at 5 -fps sampling (per frame monocular image size of $512 \times 384$ pixels). figure $3 \mathrm{c}$

\subsection{Ground-Truth Annotation of Image Data}

For each of the three experimental datasets, per image visual scene annotation (as well as labelling using numeric nomenclature) is carried out by two individuals in terms of observed objects (i.e., rocks). Both individuals use a common (planetary rock) annotation tool purposely built at the Surrey Space Centre. This results in laboriously annotated ground-truth datasets with bounding boxes encapsulating the regions of interest (i.e., rocks in the current experimental scenario) with negligible observed variation among the annotators (voiding the requirement of an interrater reliability study). These will serve as our test datasets. 


\subsection{Evaluation of Saliency Algorithm}

We test the detection performance of the aforementioned saliency algorithms using the quantitative evaluation metrics and protocols set out in [40] and [16]. We carried out an extensive quantitative evaluation of the selected saliency models for rock detection utilising datasets that replicate a planetary surface. This will enable us to understand the advantages of the different types of visual stimuli that these models use that can effectively detect rocks on planetary surfaces for rover autonomy. The analysis will moreover help in choosing the right framework on which to build the SAMS algorithm.

The detection accuracy of the proposed methods are evaluated using protocols similar to [16. For any given frame ' $t$ ', the number of 'false positives' $\left(f p_{t}\right)$, 'misses' $\left(m s_{t}\right)$ and 'true positives' $\left(t p_{t}\right)$ is calculated by measuring the spatial overlap between the ground-truth and the system output objects. If for a given frame ' $t$ ', $G_{i}^{t}$ is the $i^{t h}$ ground-truth object and $D_{i}^{t}$ is the $i^{t h}$ detected object then the spatial overlap ratio $\left(O R_{i}^{t}\right)$ is calculated as per equation 4

$$
O R_{i}^{t}=\frac{\left|G_{i}^{t} \cap D_{i}^{t}\right|}{\left|G_{i}^{t} \cup D_{i}^{t}\right|}
$$

The detected object is considered a true positive for $O R_{i}^{t} \geq 0.2$ and false positive for $O R_{i}^{t}<0.2$. Whereas any unmatched objects in the ground-truth set are considered misses. The Normalised Multiple Object Detection Accuracy (N-MODA) is computed for the entire image sequence of each dataset.

$$
N-M O D A=1-\frac{\sum_{t=1}^{N_{\text {frames }}}\left(c_{m s}\left(m s_{t}\right)+c_{f}\left(f p_{t}\right)\right)}{\sum_{t=1}^{N_{\text {frames }}} N^{t}}
$$

where

$$
N^{t}= \begin{cases}N_{G}^{t}, & \text { if } N_{G}^{t} \geq N_{D}^{t} \\ N_{D}^{t}, & \text { if } N_{G}^{t}<N_{D}^{t}\end{cases}
$$

For $\sum_{t=1}^{N_{\text {frames }}} N^{t}=0$ we force N-MODA $=0$. The parameters $c_{m s}$ and $c_{f}$ are weighting parameters that can be varied according to the specified application (in the current paper, $c_{m s}=c_{f}=1, N_{G}^{t}$ and $N_{D}^{t}$ are the number of ground-truth and system detected objects respectively. 
. The majority of the saliency methods tested here manifested good performance against the annotated ground truth (see Table 1), however two models based on the feature integration theory" (FIT), such as, STB and GBVS achieved the least performance scores in terms of N-MODA. The STB model generated local density of saliency fixations around the higher intensity pixels within the simulated rocks. Since simulated rock textures have uniformly distributed clusters of greyscale intensity pixels with hard boundaries, they act as local conspicuous regions deviating from the surrounding pixels.

It naturally attracted the proto-objects based attention towards these small areas on the rock's surface, resulting in smaller fixations which did not satisfy the evaluation criterion, causing a lot of miss detections. This anomaly is clearly noticeable from the very high miss rate and false negatives for STB. In the case of GBVS, classification of pixels as salient rocks was performed in terms of probability distributions; the simulated textures (similar to STB) of the rocks were adding a slight centre-bias towards specific regions within the rock. As a result of this behaviour, the equilibrium distribution of Markov chains selectively increased the conspicuity of the larger rocks located at the centre of the image, where the surrounding smaller rocks were given negligible weightings. This resulted in a relatively higher number of false negatives. It is worth noting however that the performance of GBVS was still better than STB in terms of N-MODA. Given the overall success of the Itti algorithm, it has been chosen as the base method to build the SAMS algorithm on since any improvement over Ittis algorithm would surely result in a better performance than the other algorithms.

\subsection{The SAMS Algorithm}

SAMS is built on a similar concept to the Wang et al model [41] where the depth map is computed as a separate process to the feature maps then combined in one final saliency map (see figure 1). However unlike the Wang et al model the generation of the depth saliency map does not require various steps and transformations to achieve a suitable shape feature map (see figure 


\begin{tabular}{|llccc|}
\hline & Dataset & N-MODA & Miss Rate & TPR \\
\hline Itti & LAB & $\mathbf{0 . 8 0}$ & 0.15 & 0.85 \\
& PANGU & $\mathbf{0 . 8 4}$ & 0.141 & 0.89 \\
\hline GBVS & SEEKER & 0.32 & 0.56 & 0.44 \\
& LAB & 0.51 & 0.43 & 0.57 \\
\hline & PANGU & 0.55 & 0.34 & 0.66 \\
\hline PQFT & SEEKER & 0.14 & 0.75 & 0.22 \\
\hline & LAB & 0.76 & 0.17 & 0.83 \\
\hline & PANGU & 0.78 & 0.04 & 0.96 \\
\hline SDSR & SEEKER & $\mathbf{0 . 4 9}$ & 0.13 & 0.87 \\
\hline & PANGU & 0.66 & 0.27 & 0.73 \\
\hline & SEEKER & 0.47 & 0.10 & 0.90 \\
\hline SigSal & LAB & 0.62 & 0.33 & 0.67 \\
\hline & PANGU & 0.78 & 0.15 & 0.85 \\
\hline & SEEKER & 0.29 & 0.51 & 0.48 \\
\hline SRA & LAB & 0.74 & 0.18 & 0.82 \\
\hline & PANGU & 0.83 & 0.01 & 0.99 \\
\hline & SEEKER & 0.45 & 0.15 & 0.85 \\
\hline & LAB & 0.49 & 0.42 & 0.58 \\
\hline & PANGU & 0.47 & 0.30 & 0.70 \\
\hline & SEEKER & 0.39 & 0.57 & 0.43 \\
\hline
\end{tabular}

Table 1: Normalized performance results for various saliency algorithms on chosen datasets. Bold numbers are highest N-MODA values within each dataset. 
2). In addition the SAMS algorithm combines all the individual feature maps 340 (structure, colour, intensity and orientation) together in one step unlike the Wang et al algorithm that computes a saliency map from 2D information then combines this map with the 3D depth map. This gives the flexibility of assigning different weights to each feature map depending on the application.

\subsubsection{Computation of shape feature map}

The SfS computation process has been developed in $\mathrm{C}++$ where it program takes an image and a parameter file to compute the scene structure, returning a Digital Terrain Model (DTM) in the form of a dense matrix with the same size of the original image. The program also attempts to recover the albedo information as described in [32. The parameter file contains information about the light direction, the maximum number of iterations allowed and a scale factor. The light direction is deduced from the rovers on-board sun sensor.

The maximum number of iteration allowed limits the number of cycles the Jacobian iterator is allowed to recapitulate in order to achieve convergence. The iteration scalar is arbitrarily set, however this has an impact on the quality of the output in terms of accuracy at the trade-off of execution speed. Since the algorithm is not performing precise calculations and is only concerned with highlighting objects that protrude from the ground, the iteration value is set to a value of 2 in order to maximise the execution speed.

The scale value also has an impact on the quality of the output and executions speed. Essentially the scale value is used to resize the image to smaller dimensions producing a coarser DTM but a much faster execution. Once again, since the algorithm is not performing precise calculations, the Scale value is set to reduce the image size to $320 \times 240$ pixels or as close to this as possible depending on the image shape. The resulting DTM is the scaled back to match the size of the original image and a Gaussian filter is applied to smooth out any noise. The typical execution speed for generating a DTM using the above parameters is in the region of 0.2 seconds on a Raspberry Pi Type B with CPU speed of 700Mhz. 


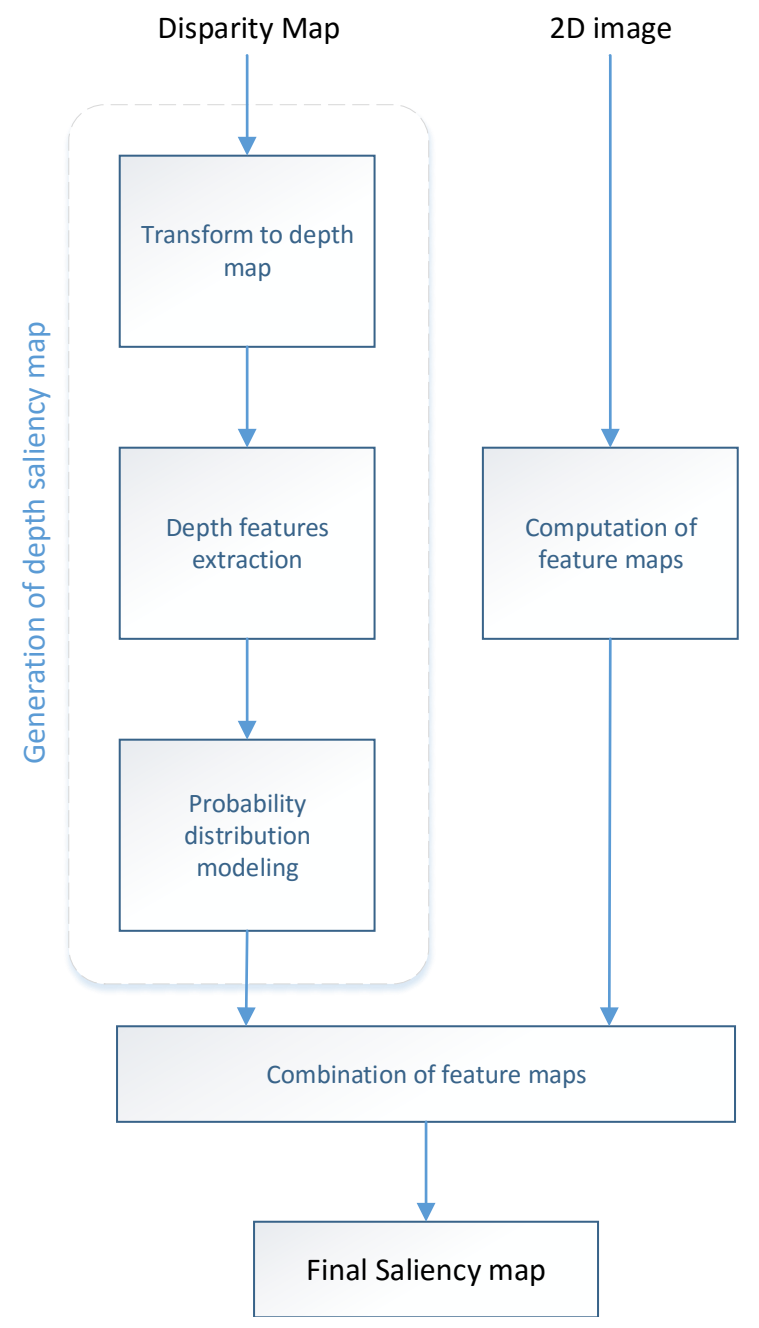

Figure 1: Depth saliency model as proposed by Wang et al [41. 


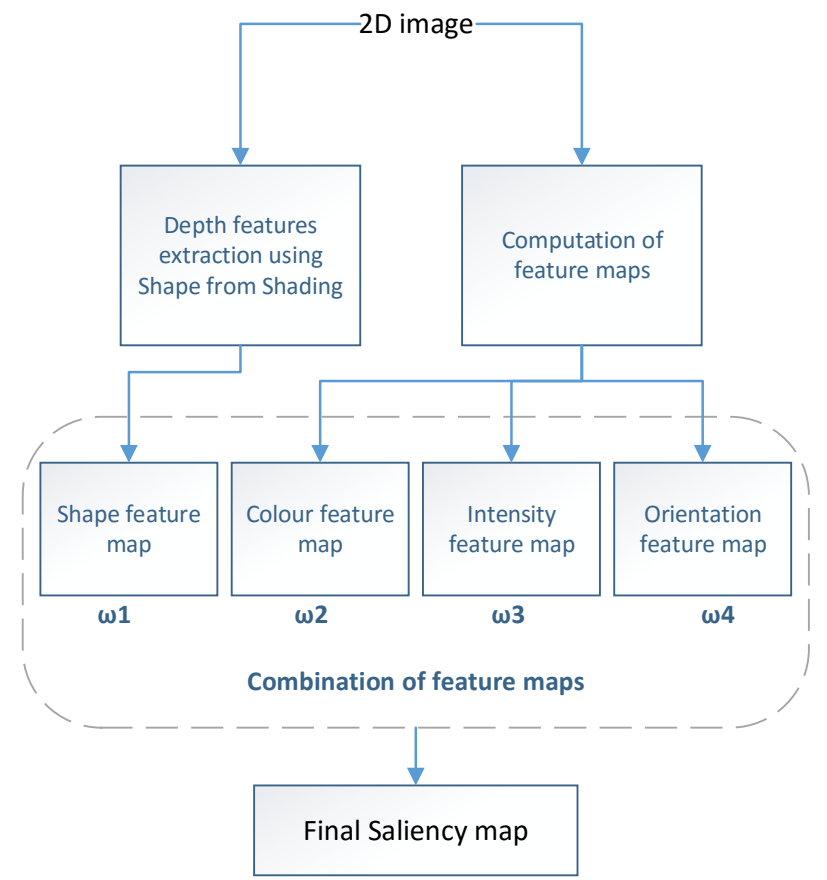

Figure 2: Proposed Shape Augmented Monocular Saliency model. 


\subsubsection{Computation of feature maps} centre surround difference method proposed by Itti et al [7, however the algorithm falls short of combining the feature maps together at this stage.

\subsubsection{Combining the feature maps}

Several approaches of combining feature maps have been proposed in literature, however, a standardized approach to combine depth maps with $2 \mathrm{D}$ visual features is still lacking. In [41] the proposed model is to adopt a weighted merging mechanism of the feature maps where the final saliency map is equal to the sum of both maps as in equation 6 .

$$
S M_{s}(i, j)=\omega_{1} S M_{d e p}+\omega_{2} S M_{2 D}
$$

where $\omega_{1}=\omega_{2}=0.5$.

Similarly, the SAMS algorithm performs a weighted merging of all the feature maps, however rather than combining the 2D saliency map with the depth map, the SAMS algorithm combines all 5 maps together as per equation 7 .

$$
S M_{F}(i, j)=\omega_{1} F M_{S}+\omega_{2} F M_{C}+\omega_{3} F M_{I}+\omega_{4} F M_{O}
$$

where $\omega_{n}=1 / \# F M$ and $F M_{m}$ is the feature map with $S$ for the Structure (or depth) feature map, $C$ for the colour feature map, $I$ for the intensity feature map and $O$ for the orientation feature map.

Experiments performed using various weights for each feature map are analogous with the experiments perform in 41 in that equal weighting on all the feature maps tend to yield the best detection performance. Table 2 highlights some of the weight values and their N-MODA, miss rate and true positives detected by the SAMS algorithm using the Lab dataset.

Performance evaluations for SAMS versus Itti saliency-based algorithms are shown in Table 3. Results indicate that the SAMS algorithm performs better than the standard Itti algorithm over all datasets. It is worth reiterating that 


\begin{tabular}{cccc|ccc}
$\omega_{1}$ & $\omega_{2}$ & $\omega_{3}$ & $\omega_{4}$ & N-MODA & Miss Rate & True Positives \\
0.25 & 0.25 & 0.25 & 0.25 & $\mathbf{8 3 . 6 3}$ & $\mathbf{1 1 . 2 6}$ & $\mathbf{8 8 . 7 4}$ \\
0.4 & 0.2 & 0.2 & 0.2 & 80.27 & 17.12 & 82.88 \\
0.2 & 0.4 & 0.2 & 0.2 & 76.38 & 22.77 & 77.23 \\
0.2 & 0.2 & 0.4 & 0.2 & 81.94 & 19.44 & 80.56 \\
0.2 & 0.2 & 0.2 & 0.4 & 73.21 & 24.88 & 75.12
\end{tabular}

Table 2: Feature map weights and their results in \%. Bold numbers indicate best results.

\begin{tabular}{l|ccc|ccc} 
Dataset & \multicolumn{3}{|c|}{ Itti Saliency Algorithm } & \multicolumn{3}{c}{ SAMS Algorithm } \\
\hline & N-MODA & Miss Rate & True Positives & N-MODA & Miss Rate & True Positives \\
LAB-based & 80.23 & 15.10 & 84.90 & 83.63 & 11.26 & 88.74 \\
PANGU & 84.11 & 11.27 & 88.73 & 87.60 & 7.89 & 92.11 \\
SEEKER & 31.85 & 56.32 & 43.68 & 80.90 & 17.23 & 82.77
\end{tabular}

Table 3: Performance evaluation between the Itti and the SAMS algorithms expressed as \%.

this performance is achieved in purely unsupervised manner, without any topdown feedback or any a-priori knowledge of the test datasets used.

Although the improvement for N-MODA, Miss Rate and True Positives are marginal for the LAB- based and PANGU datasets, there is a noticeable increase for the SEEKER dataset. This is due to the additional dimension of information introduced by the structure feature map and noise reduction on the other maps when the same structure map is added to the other feature maps. This can be clearly seen in the figures 45 and 6 . The Colour feature map has been omitted since the images are in grayscale.

The output of the SAMS algorithm is much easier to threshold using Otsus bimodal distribution as the salient features are quite prominent with very little false positives that are detected as salient features.

Figure 6 shows the result of the bimodal distribution thresholding of both outputs and shows bounding boxes overlaid on the original image. The blue bounding boxes in figure 6 show the salient objects detected by the Itti algo- 


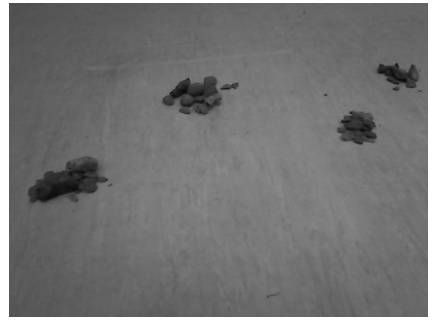

(a)

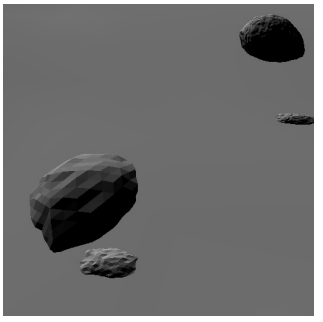

(b)

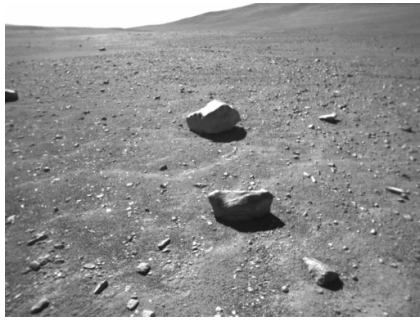

(c)

Figure 3: Example images from the Lab 3a PANGU simulated 3b and SEEKER 3c datasets.

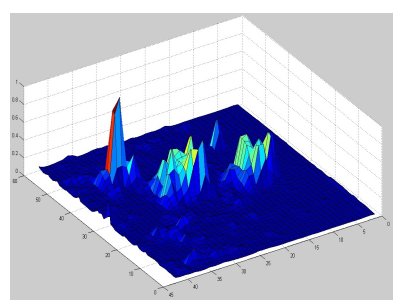

(a)

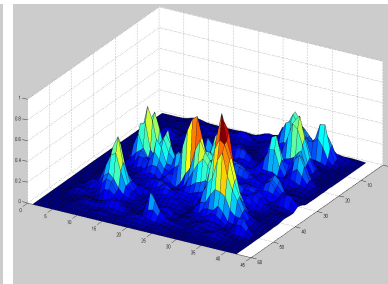

(b)

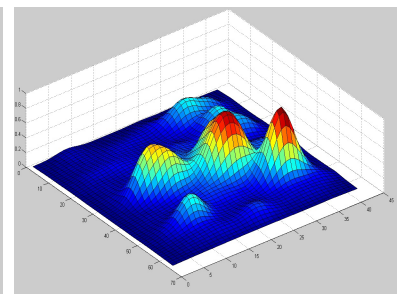

(c)

Figure 4: Itti algorithm feature maps $4 \mathrm{a}=$ Intensity, $4 \mathrm{~b}=$ Orientation and $4 \mathrm{c}=$ Final output.

rithm. The algorithm has detected numerous features that should not have been classified as salient. In addition, if these small features were to be considered as a salient object, the algorithm fails to detect similar objects dotted around the image. Furthermore, the actual salient objects (large rocks) have been detected as one whole feature rather than 3 different objects.

The red bounding boxes in figure 6 show the salient objects detected by the SAMS algorithm. The algorithm ignores very small features that would not have any significant value in terms of providing information for planetary exploration. The output of the SAMS algorithm closely correlates with the human annotated objects with minor variations in the size of the bounding boxes.

In an effort to verify that the algorithm performs well in a real world application, a small selection of images from the vast MER libraries where tested using both methods. Figure 7 a to e show the results where bounding boxes in red are the salient objects detected by the SAMS algorithm while bounding 


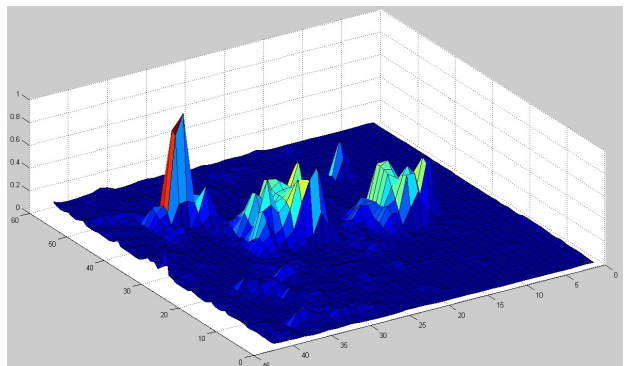

(a)

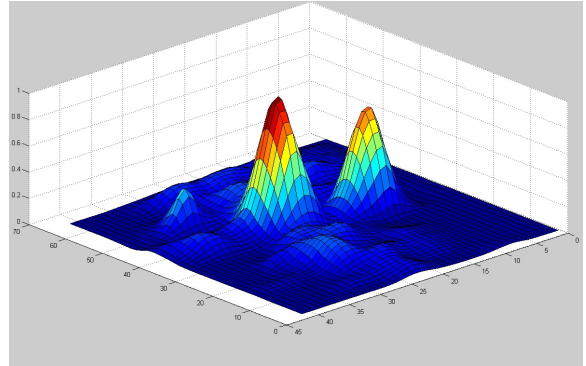

(c)

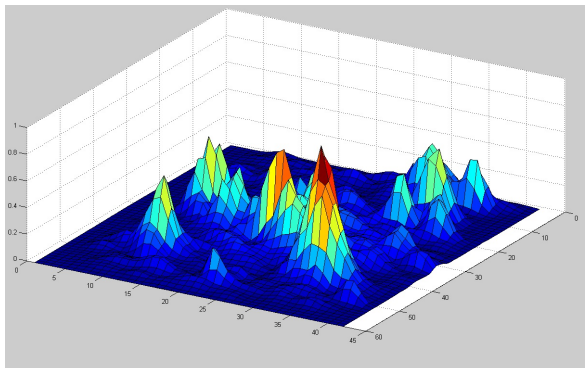

(b)

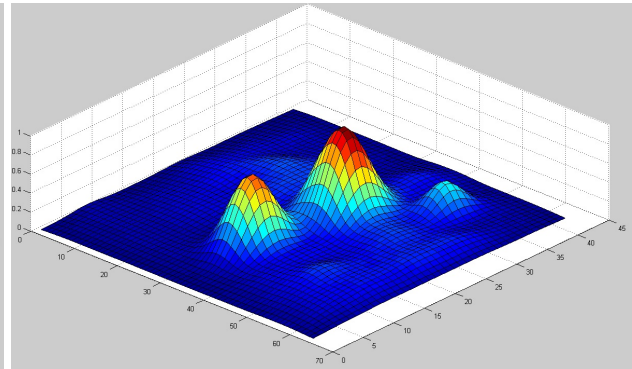

(d)

Figure 5: Feature maps for SAMS algorithm where $5 \mathrm{a}=$ Intensity, $5 \mathrm{~b}=$ Orientation, $5 \mathrm{c}=$ Structure and $5 \mathrm{~d}=$ Final output.

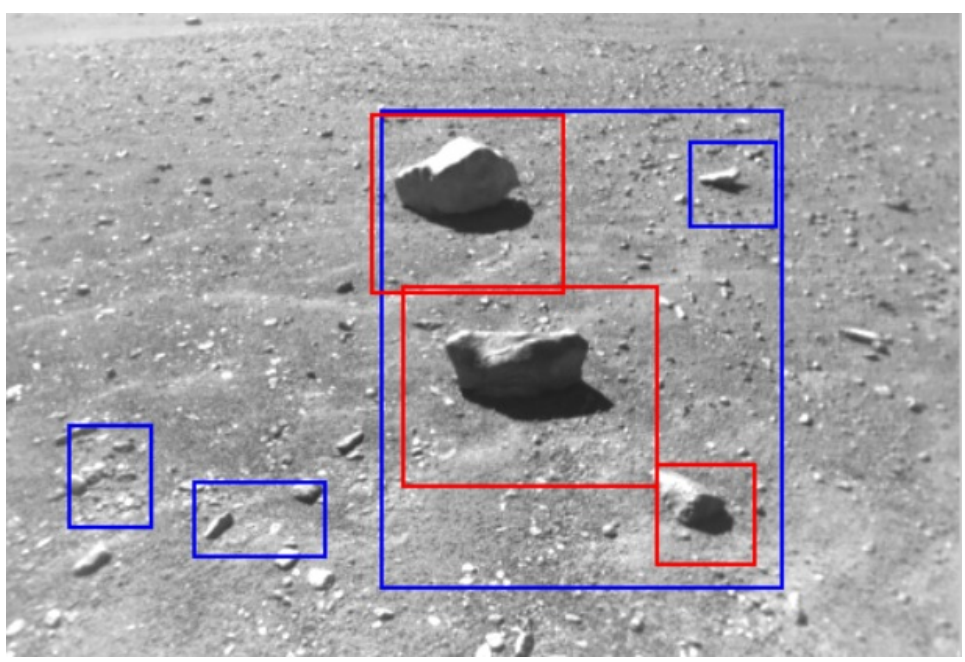

Figure 6: Result of thresholding the final feature maps for both algorithms. Blue boxes belong to the Itti algorithm while Red boxes belong to the SAMS algorithm. 


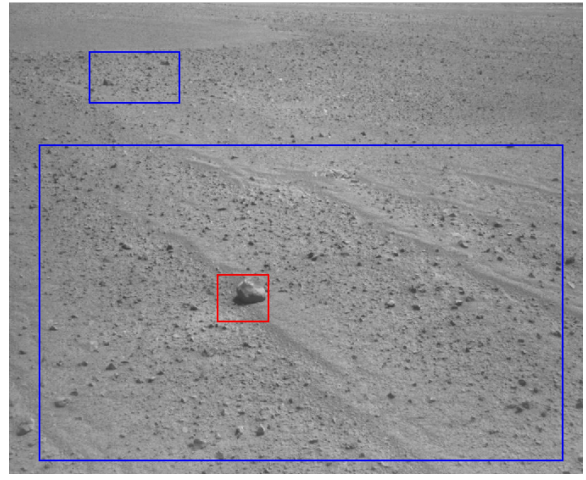

(a)

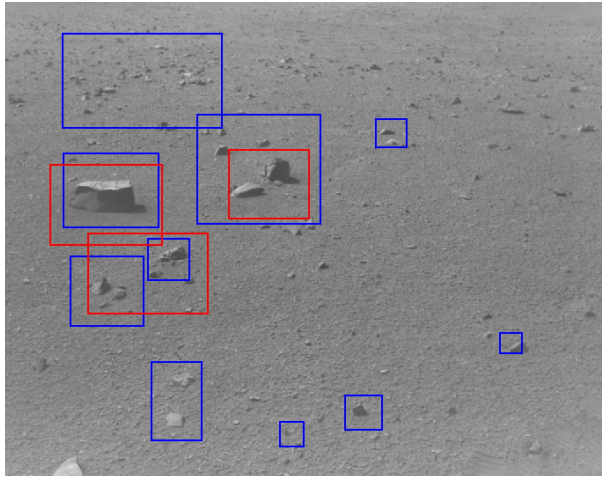

(b)

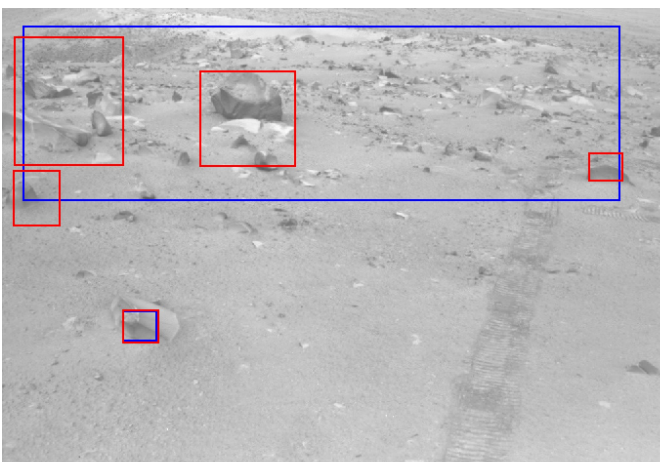

(c)

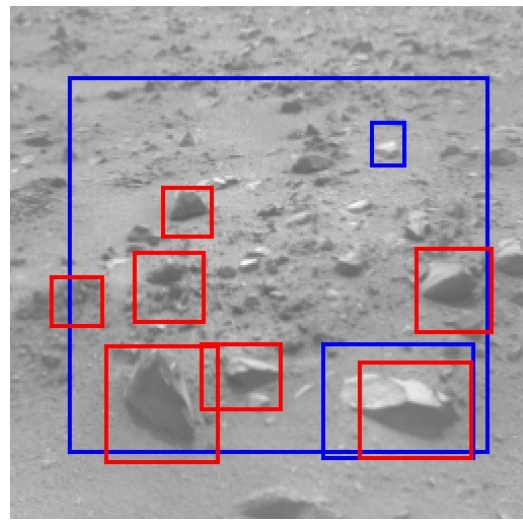

(d)

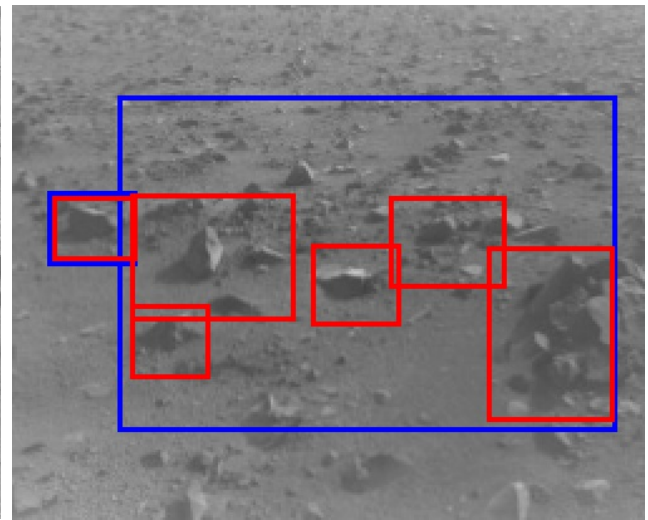

(e)

Figure 7: Salient object detection on MER images using the SAMS (Red bounding boxes) and Itti (Blue bounding boxes) algorithms. Orignal image names within JPL's PDS image repository are $7 \mathrm{a}=1 \mathrm{n} 436950889 \mathrm{fflc} 9 \mathrm{~h} 1 \mathrm{p} 0723 \mathrm{r} 0 \mathrm{~m} 1 . \mathrm{mg}, 7 \mathrm{~b}=1 \mathrm{n} 463314413 \mathrm{fflchj3p} 196610 \mathrm{~m} 1$.img, $7 \mathrm{c}=1 \mathrm{n} 468107164$ fflcibnp1825r0m1.img, 7d = 1n467395302dnlch0uf000610m1.img, 7d = 1n466780272dnlchzof000610m1.img 
boxes in blue are salient objects according to the Itti algorithm. It is evident that the SAMS algorithm outperforms the Itti algorithm where the SAMS algorithm is able to identify individual objects (rocks) in complex scenes rather than detect an aggregation of objects. The SAMS algorithm also omits small objects in the scene that can hardly be considered as salient when much larger objects are present. It is worth reiterating that both algorithms use the same Otsu binerization method with identical parameters.

\subsection{Computation time}

Finally we set out to test the Saliency map computation time (averaged over the total number of frames) which is computed for all models using the whole set of datasets in order to find out which of these models would have the lowest computation time within the current problem domain (refer to figure 8). The experimental workstation used for generating these results comprises a quadcore Intel(R) Core(TM) i5-2400 CPU (3.10 GHz) running Linux (Ubuntu10.04, 64-bit architecture). The SAMS algorithm is also benchmarked against these results.

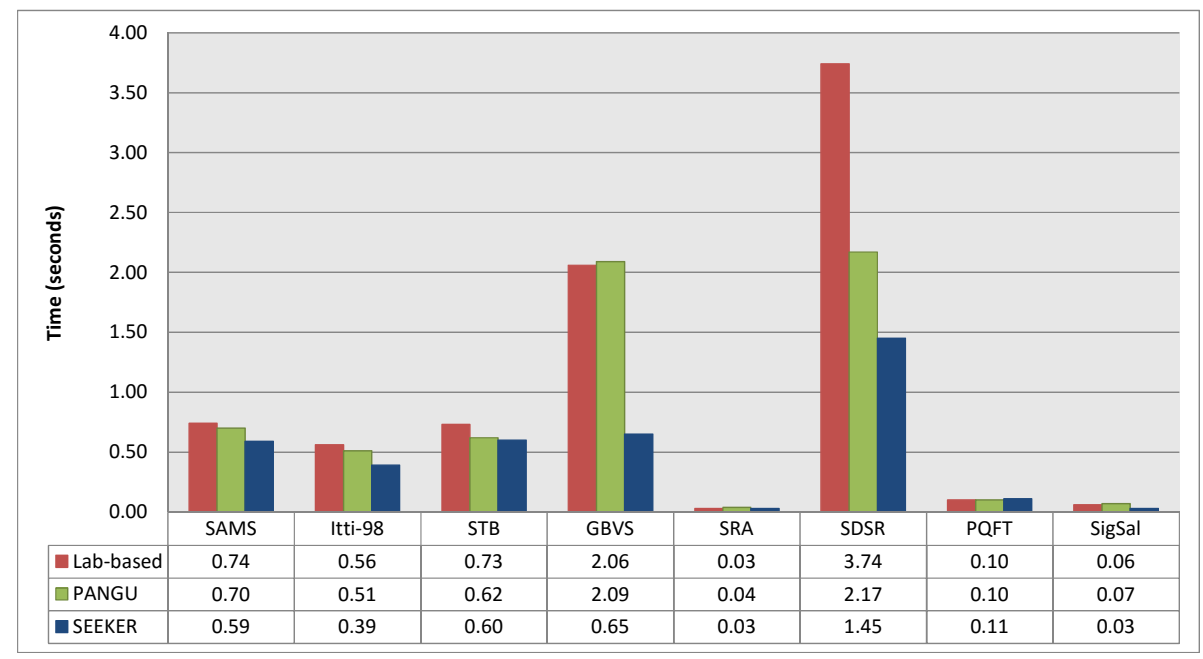

Figure 8: Computation time of the various algorithms for each dataset. 
As depicted in figure 8, SDSR took the longest time to process an image, whereas SigSal, PQFT and SRA required the lowest processing time. The Itti algorithm performs relatively well in terms of computational speed however it is slower than the 3 aforementioned algorithms. Since SAMS is built on the Itti algorithm the computation speed is slightly slower due to the extra computational requirements to generate the DTM however its performance is still at an acceptable rate and this can be improved using parallel processing methods for generating the DTM as described in 31.

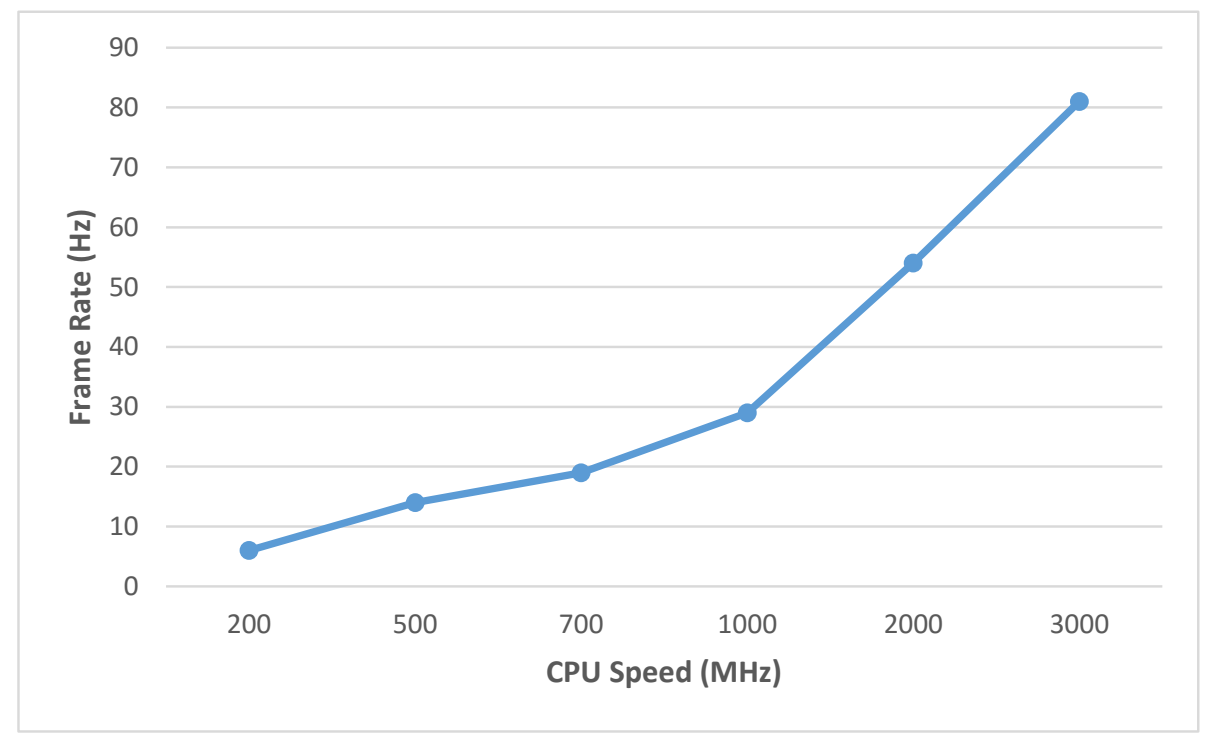

Figure 9: SAMS algorithm frame rate for various CPU speeds.

Furthermore, the algorithm was run on a virtual machine Linux distribution with a single $2 \mathrm{GHz}$ virtual $\mathrm{CPU}$ core and $512 \mathrm{Mb}$ of virtual RAM. The $\mathrm{CPU}$ time of the virtual core was throttled down to simulate various CPU speeds as found on On-Board Computers (OBC) of explorations rovers. The speeds are: $200 \mathrm{MHz}, 500 \mathrm{Mhz}, 700 \mathrm{Mhz}, 1 \mathrm{Ghz}$, and $2 \mathrm{Ghz}$ and the corresponding frame rate of our algorithm is shown in figure 9 The SEEKER dataset has been used for this experiment and the images have been scalled down by a factor of 4 with the resulting image size of $128 \times 96$ pixels. This has little or no degradation in 
results measured using the N-MODA metric. It is worth noting that the MSL rover has an $\mathrm{OBC}$ with $200 \mathrm{MHz}$ and that the Raspberry Pi (an inexpensive computer) has a CPU speed of $700 \mathrm{Mhz}$, both of which would be capable of executing the SAMS algorithm.

\section{Conclusion}

We propose a novel approach towards detecting salient objects with specific focus of application within the domain of planetary rovers. The algorithm (SAMS) proposed in this paper uses photoclinometry to add a shape dimension to the feature maps employed by saliency algorithms such as the Itti et al method. This greatly reduces the computational costs required by $3 \mathrm{D}$ saliency algorithms when constructing the DTM through a stereo pair of images. Results thus achieved showed good performance over a previous study using the same datasets and annotations. Such paradigms could potentially form a very effective basis for object detection specifically for applications in future longdistance autonomous rover navigation. We also anticipate exploring many other novel dimensions for the SAMS algorithm and experiment with more challenging datasets to achieve a solid foundation for the proposed concept.

Field trails at a location that mimics a Martian or Lunar terrain is being planned using the Surrey Mobility Platform (SMP) Rover. This platform has been successfully utilized in previous field trial as described in [27.

\section{Acknowledgement}

The authors are very grateful to the Autonomous Systems Group of RAL Space for providing the SEEKER dataset. 


\section{References}

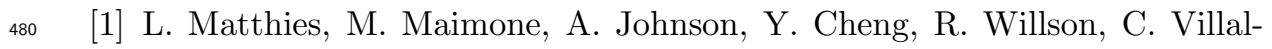
pando, S. Goldberg, A. Huertas, A. Stein, A. Angelova, Computer vision on mars, International Journal of Computer Vision.

[2] S. Hrabar, G. Sukhatme, Vision-based navigation through urban canyons, Journal of Field Robotics 26 (5) (2009) 431-452. doi:10.1002/rob. 20284 URL http://dx.doi.org/10.1002/rob.20284

[3] R. Hadsell, P. Sermanet, J. Ben, A. Erkan, M. Scoffier, K. Kavukcuoglu, U. Muller, Y. LeCun, Learning long-range vision for autonomous off-road driving, J. Field Robot. 26 (2) (2009) 120-144. doi:10.1002/rob.v26:2. URL http://dx.doi.org/10.1002/rob.v26:2 sification using three-dimensional ladar data for ground robot mobility, Journal of Field Robotics 23 (10) (2006) 839 - 861.

[ [5] Y. Gao, C. Spiteri, M.-T. Pham, S. Al-Milli, A survey on recent object a detection techniques useful for monocular vision-based planetary terrain classification, Robotics and Autonomous Systems 62 (2) (2014) 151 - 167. doi:http://dx.doi.org/10.1016/j.robot.2013.11.003

口 URL http://www.sciencedirect.com/science/article/pii/ S0921889013002200

[6] A. Borji, D. N. Sihite, L. Itti, Quantitative analysis of human-model agreement in visual saliency modeling: A comparative study, Image Processing, IEEE Transactions on 22 (1) (2013) 55-69.

[7] L. Itti, C. Koch, E. Niebur, A model of saliency-based visual attention for rapid scene analysis, IEEE Transactions on Pattern Analysis and Machine Intelligence 20 (11) (1998) 1254-1259. doi:10.1109/34.730558. 
[8] A. Shaukat, C. Spiteri, Y. Gao, S. Al-Milli, A. Bajpai, Quasi-thematic feature detection and tracking for future rover long-distance autonomous navigation, Proc. ESA Conference on Advanced Space Technologies in Robotics and Automation, ASTRA, Noordwijk, Netherlands.

[9] A. Bajpai, G. Burroughes, A. Shaukat, Y. Gao, Planetary monocular simultaneous localization and mapping, Journal of Field Robotics 33 (2) (2016) 229-242.

[10] Y. Gao, Contemporary Planetary Robotics: An Approach Toward Autonomous Systems, John Wiley \& Sons ISBN: 978-3-527-41325-6, 2016.

[11] C. Liu, P. C. Yuen, G. Qiu, Object motion detection using information theoretic spatio-temporal saliency, Pattern Recognition 42 (11) (2009) 2897 - 2906. doi:http://dx.doi.org/10.1016/j.patcog.2009.02.002. URL http://www.sciencedirect.com/science/article/pii/ S0031320309000685

[12] J. Yan, M. Zhu, H. Liu, Y. Liu, Visual saliency detection via sparsity 520 \ pursuit, IEEE Signal Processing Letters 17 (8) (2010) 739-742. doi:10. 1109/LSP. 2010.2053200.

[13] C. Siagian, L. Itti, Biologically inspired mobile robot vision localization, (1. IEEE Transactions on Robotics 25 (4) (2009) 861-873. doi:10.1109/TRO. 2009.2022424

[14] M. Hayhoe, D. Ballard, Eye movements in natural behavior, Trends in cognitive sciences 9 (4) (2005) 188-194.

[15] D. Walther, C. Koch, Modeling attention to salient proto-objects, Neural Networks 19 (9) (2006) 1395-1407. doi:10.1016/j.neunet.2006.10.001. URL http://dx.doi.org/10.1016/j.neunet.2006.10.001

[16] R. Kasturi, D. Goldgof, P. Soundararajan, V. Manohar, J. Garofolo, R. Bowers, M. Boonstra, V. Korzhova, J. Zhang, Framework for performance evaluation of face, text, and vehicle detection and tracking in video: 
Data, metrics, and protocol, IEEE Transactions on Pattern Analysis and Machine Intelligence 31 (2) (2009) 319-336. doi:10.1109/TPAMI.2008.57

[17] S. Suzuki, K. be, Topological structural analysis of digitized binary images by border following, Computer Vision, Graph-

n ics, and Image Processing 30 (1) (1985) 32 - 46. doi:http: //dx.doi.org/10.1016/0734-189X (85)90016-7.

a URL http://www.sciencedirect.com/science/article/pii/ 0734189X85900167

[18] J. Harel, C. Koch, P. Perona, Graph-based visual saliency., in: B. Schlkopf, J. C. Platt, T. Hofmann (Eds.), NIPS, MIT Press, 2006, pp. 545-552.

a URL http://dblp.uni-trier.de/db/conf/nips/nips2006.html\# HarelKP06

[19] X. Hou, L. Zhang, Saliency detection: A spectral residual approach, in: In IEEE Conference on Computer Vision and Pattern Recognition (CVPR07). IEEE Computer Society, 2007, pp. 1-8.

[20] H. J. Seo, P. Milanfar, Static and space-time visual saliency detection

n by self-resemblance, Journal of Vision 9 (12) (2009) 15. arXiv:/data/ Journals/JOV/932859/jov-9-12-15.pdf, doi :10.1167/9.12.15.

URL +http://dx.doi .org/10.1167/9.12.15

[21] C. Guo, L. Zhang, A novel multiresolution spatiotemporal saliency detection model and its applications in image and video compression, IEEE Transactions on Image Processing 19 (1) (2010) 185 - 198. doi:10.1109/ TIP.2009.2030969.

[22] X. Hou, J. Harel, C. Koch, Image signature: Highlighting sparse salient regions, IEEE Transactions on Pattern Analysis and Machine Intelligence 34 (1) (2012) 194-201. doi:10.1109/TPAMI.2011.146.

[23] C. Koch, S. Ullman, Shifts in selective visual attention: towards the underlying neural circuitry, Human Neurobiology 4 (1985) 219-227. 
[24] A. Saxena, M. Sun, A. Y. Ng, Make3d: Learning 3d scene structure from a single still image, IEEE Trans. Pattern Anal. Mach. Intell. 31 (5) (2009) 824-840. doi:10.1109/TPAMI.2008.132.

URL http://dx.doi.org/10.1109/TPAMI .2008.132

[25] A. Saxena, S. H. Chung, A. Y. Ng, 3-d depth reconstruction from a single still image, International Journal of Computer Vision (IJCV 76 (2007) 2007.

[26] D. Hoiem, A. A. Efros, M. Hebert, Recovering surface layout from an image, International Journal of Computer Vision 75 (1) (2007) 151-172. doi: 10.1007/s11263-006-0031-y. URL http://dx.doi .org/10.1007/s11263-006-0031-y

[27] C. Spiteri, S. Al-Milli, Y. Gao, A. S. de Len, Real-time visual sinkage detection for planetary rovers, Robotics and Autonomous Systems 72 (2015) 307 - 317. doi:http://dx.doi.org/10.1016/j.robot.2015.06.009

口 URL http://ww.sciencedirect.com/science/article/pii/ S0921889015001372

[28] F. Irom, G. Swift, F. Farmanesh, A. Johnston, Single-event upset in commercial silicon-on-insulator powerpc microprocessors, in: SOI Conference,

口 IEEE International 2002, 2002, pp. 203-204. doi:10.1109/SOI.2002. 1044476

[29] R. Lindemann, C. Voorhees, Mars exploration rover mobility assembly design, test and performance, in: Systems, Man and Cybernetics, 2005 IEEE International Conference on, Vol. 1, 2005, pp. 450-455 Vol. 1. doi:10.1109/ICSMC. 2005.1571187

[30] J. van Diggelen, A photometric investigation of slopes and heights of the ranges in maria of the moon, Bulletin of the Astronomical Institutes of the Netherlands 11 (1951) 283.

[31] Shape from shading using linear approximation. 
[32] P.-S. Tsai, M. Shah, Shape from shading with variable albedo, Optical Engineering 37 (4) (1998) 1212-1220. doi:10.1117/1.601957.

URL http://dx.doi.org/10.1117/1.601957

[33] D. E. A. Wilhelms, Summary of lunar stratigraphy - Telescopic observations - Contributions to Astrogeology, Geological Survey Professional Paper; 599F, Washington, D.C. : U.S. Geological Survey, 1970, prepared on behalf of the National Aeronautics and Space Administration.

[34] M. Taguchi, G. Funabashi, S. Watanabe, Y. Takahashi, H. Fukunishi, Lunar albedo at hydrogen lyman $\alpha$ by the nozomi/uvs, Earth, Planets and Space 52 (9) (2014) 645-647. doi:10.1186/BF03351673.

URL http://dx.doi.org/10.1186/BF03351673

[35] S. Biswas, G. Aggarwal, R. Chellappa, Robust estimation of albedo for illumination-invariant matching and shape recovery., in: ICCV, IEEE Computer Society, 2007, pp. 1-8.

a URL http://dblp.uni-trier.de/db/conf/iccv/iccv2007.html\# BiswasAC07

[36] R. Zhang, P.-S. Tsai, J. E. Cryer, M. Shah, Shape from shading: A survey, IEEE TRANSACTIONS ON PATTERN ANALYSIS AND MACHINE INTELLIGENCE 21 (8) (1999) 690-706.

[37] V. S. Ramachandran, Perceiving Shape From Shading, Scientific American $256(8)$.

[38] N. Otsu, A Threshold Selection Method from Gray-level Histograms, IEEE

Transactions on Systems, Man and Cybernetics 9 (1) (1979) 62-66. doi: 10.1109/TSMC.1979.4310076.

URL http://dx.doi.org/10.1109/TSMC.1979.4310076

[39] Y. Zheng, J. Yu, S. B. Kang, S. Lin, C. Kambhamettu, Single-image vignetting correction using radial gradient symmetry., in: CVPR, IEEE Computer Society, 2008. 
URL http://dblp.uni-trier.de/db/conf/cvpr/cvpr2008.html\# ZhengYKLK08

[40] J. C. Nascimento, J. S. Marques, Performance evaluation of object detection algorithms for video surveillance, IEEE Transactions on Multimedia 8 (4) (2006) 761-774. doi:10.1109/TMM.2006.876287.

[41] J. Wang, M. P. D. Silva, P. L. Callet, V. Ricordel, Computational model of stereoscopic 3d visual saliency, IEEE Transactions on Image Processing 22 (6) (2013) 2151-2165. doi:10.1109/TIP. 2013.2246176. 